\title{
Research on Integration of Digital Graphic and Information in Building Information Modeling
}

\author{
Weibo YIN ${ }^{1,2}$, Shangwei LIU ${ }^{1,3}$, Qun WEI ${ }^{1,2}$, Jun XIAO ${ }^{1, \text { a }}$ \\ ${ }^{1}$ University of Chinese Academy of Sciences, Beijing, 100049, China; \\ ${ }^{2}$ Steel Structure and Engineering Research Institute, North China University of Water Resources \\ and Electric Power, Zhengzhou, 450045, China; \\ ${ }^{3}$ School of Water Conservancy, North China University of Water Resources and Electric Power, \\ Zhengzhou, 450045, China;
}

aemail:xiaojun@ucas.ac.cn

Keywords: Digital graphic medium; Digital graphic; Information; Integration system

\begin{abstract}
The currently popular Building Information Modeling technology is substantively a dynamic integration system between digital graphics and related information of structure engineering, where information can be updated with change of graphics. The concept, construction method, description standard, basic theory and connotation of digital graphic medium are proposed in this paper, which forms corresponding projection and simulation patterns of the studied engineering object in the natural space and computer space, making the "graphics" become 5 dimensional information carrier with dynamic changing geometric and non-graphic attributes. In engineering structure described by this information system of digital graphics, data can be obtained directly from the graphics and feedback in all stages including the design, analysis, manufacture and installation, which presents the characteristics of big data. This is the advantage of BIM technology, and the reason it is achieving remarkable results in engineering applications.
\end{abstract}

\section{Introduction}

BIM is short for Building Information Modeling. BIM is based on three-dimensional digital technology. It integrates all digital databases of related information into shared core files, provides digital representation of the shape, physical and functional characteristics of a project, shares the working processes reliable for decisions and optimizations, collaborates to insert information into, extract from, update and change the database, and enhances productivity and data consistency for the parties involved in all stages. BIM has the benefits of visualization, coordination, simulation, optimization and drawing exportation.

\section{BIM Core Technology}

BIM shares information in its central database model. This information model is parameter-driven, has diversified information, unified standard and achieves collaboration. Of which parameterization is the key technology, unified standard is the core, diversified information is the support, and collaboration is the result. The current study showed only the geometric properties of graphics, and they are only displayed on the computer screen as final visual products, while their physical attributes, topology and other information are not integrated. Therefore, the concept, construction method, description standard, basic theory and connotation of digital graphic medium [1-3] are proposed in this paper, which forms corresponding projection and simulation patterns of the studied engineering object in the natural space and computer space, making the "graphics" become 5-D information carrier with dynamic changing geometric and non-graphic attributes. In engineering structure described by digital graphic medium, physical equations of nature are complied with, the studied motion and deformation are real-time simulated in a virtual environment, and remarkable results are achieved and verified in BIM projects.

The digital model for digital graphic medium is not relevant to the platform. Models of any 3D 
modeling software can be converted and integrated into digital models of this system.

Digital graphic becomes medium with both physical and geometric attributes, and engineering system of digital graphics and information is constructed; with introduced and developed CIS/2 and IFC standards, "data is attached to graphics, and graphics are containing data"; the concept of graphic developed into a 5D object. The visualized and non-graphic attributes of various components and parts of a complicated structure are integrated into graphics, making themselves contain five-dimensional space information of the spatial coordinates $(x, y, z)$, time coordinate $(t)$ and non-geometric information (v). The engineering drawings become a carrier for real-time status and relevant information of the structure; individual graphic elements with unique identifiers are also bi-directionally associated with a huge engineering database. This new digital graphic information system is presenting CAD drawings with unique technology of data storage and linkage as well as a new data exchange format; it is a key technology with significance for the developing 3-D design platforms [2-3].

It is featured by the following points:

(1) The concept, construction method, description standard, basic theory and connotation of digital graphic medium are proposed in this paper, which forms corresponding projection and simulation patterns of the studied engineering object in the natural space and computer space, making the "graphics" become 5-D information carrier with dynamic changing geometric and non-graphic attributes.

(2) Definition of geometric and non-geometric attributes and data formats for objects in natural space are proposed according to CIS/2 and IFC standards; these are standards based and data-sharing platform for the construction of digital graphic medium models.

(3) The concept and storage method of models for section key points of complicated structure drawings and skeleton grid of key point connections are proposed.

(4) Geometric information associated with the time parameters, together with material properties, temperature, stress, deformation, seepage, safety factors and other non-geometric properties are all saved into the graphics; they are also subject to dynamic adjustment and correction. The above core technology utilizes graphics as the medium, supports the main framework and fills in the contents of digital engineering; it is an integrated digital graphic information system.

\section{Standards for the Theory of Digital Graphic Medium}

CIS/2 standards are introduced into China by the authors and their teams, and appropriate standard narrative format is developed; through the definition of 360 schema, all calculation models, analysis models and manufacture models related to engineering are subject to unified description, and strict rules and description for definition, properties, classification, continuity, genetics and association of multiple models are made; This brings about unified expression method for engineering information of a same project in different departments, different professions and different periods, as well as facilitated data exchange between different applications in architecture and data management throughout the life-cycle of a building; In addition, data exchange and comparison between different projects are provided with a sharing platform.

CIS/2 is mainly used for the design, analysis and manufacture of steel structures, while IFC covers the construction works. Description for underground works in engineering requires GML language standards as the supplement, which will be discussed in other articles.

\section{Parametric BIM Family Library}

Family is a group that contains common parameter set and related graphical elements. Family variants are called family types. In BIM all graphical elements are based on family [4-7]. Family is a concept with great function in BIM. It helps for easy management of data and amendment to each graphical element; multiple types can be defined, and according to the design by its creator, each type may have different dimensions, shapes, material setup or other parameter variables.

The data required for steel structure parametric family library based on digital graphic medium 
are mainly referred to AISC profile standards, Chinese GB profile standards and other popular industrial standards for civil works and bridges, including 3660 AISC profiles, 4411 Chinese GB profiles, 75 parametric profiles, 1500 fasteners, 63 profiles for hydraulic structures, 6 standards for bridge engineering; In addition, the library also includes table data for structural mechanics properties (such as axial compression stability coefficient $\varphi$ of members, length calculation coefficient of columns, specifications and profile properties of steel, properties of combined interface, specifications and size of fasteners, designed capacity and bearing torque of members, design value of carrying capacity of connections, shear moment calculation formula for beam end and single span portal frame), cranes technical information, welding lacerations, structural assembly and installation deviations. Database and data sheets are dynamically created on the interface of database by the use of Microsoft ActiveX Data Objects Library (ADO) and Microsoft ADO Extension 2.1 for DDL Security (ADOX).

ADO and ADOX are quoted in the running program to create database and data sheets dynamically. With the help of ADO node sheet, the user can search for interested profiles (see Fig.1), or choose to generate 2-D views or 3-D models as desired. In addition, the software system provides customized profiles being extensible into 3-D modeling of concrete, reinforced concrete and modern complicated engineering works.

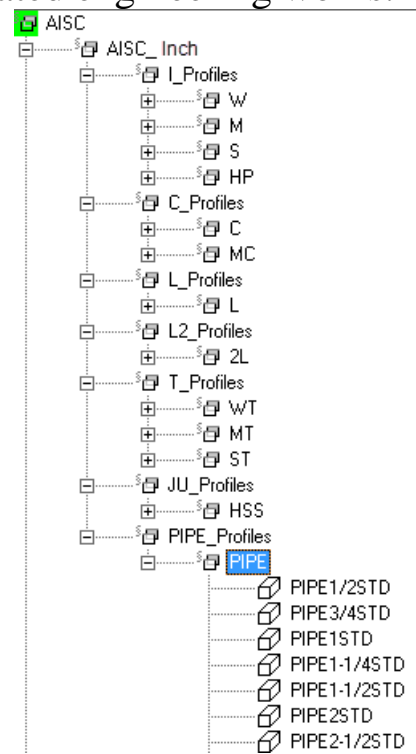

(a)AISC profile database

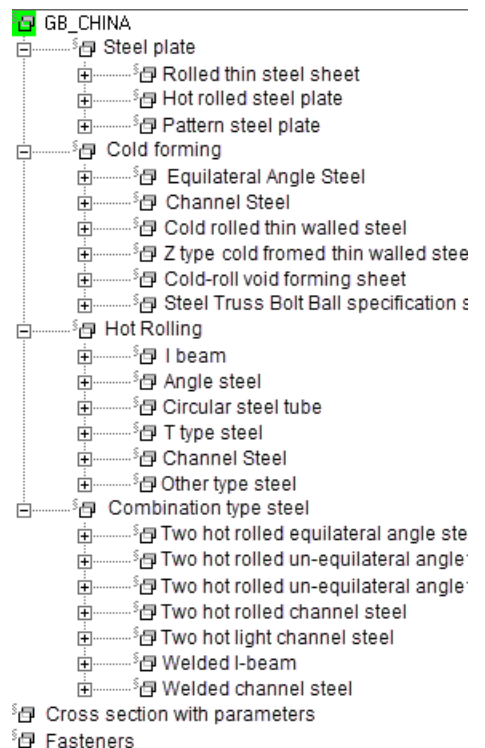

(b) Chinese GB profile database

Fig.1 Common profile database

Parametric family of graphic database provides an effective method for 3D modeling and properties dynamic recording, which has significance in improvement of modeling accuracy, speed and quality.

\section{BIM Application on Modeling}

By the use of the theory and method of digital graphic medium, the authors and their teams developed the CIS2CAD software which is based on CIS/2 standard formats $(400,000$ sentences, with independent copyright). This software can directly read, design, analyze and produce models' stp files, and generate in real time the 3-D CAD model for the entire project; The corresponding Access database will generate virtual reality vrml files, and automatically generate detailed construction drawings and workshop drawings; graphic editing tools are also provided. Fig.2-4 is a brief introduction to the development and application of CIS2CAD software [6-7]. 


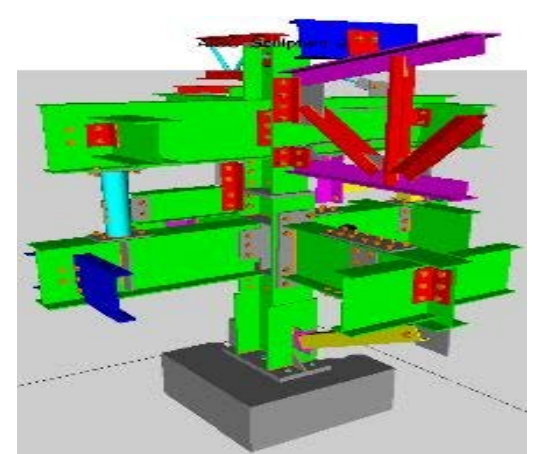

Fig.2 Steel structure, from SDS/2

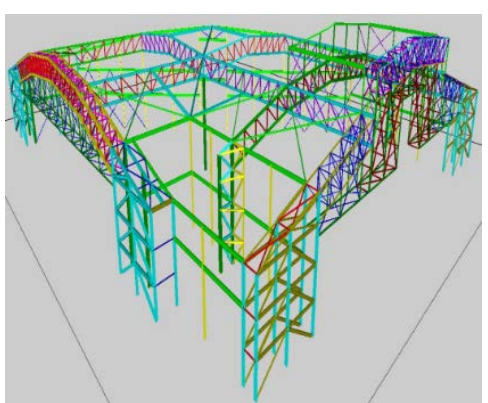

Fig.3 Steel structure, from GT STRUDL

\section{BIM Application on Construction}

For construction works in 3-D space, BIM can (1) provide information related to construction quality, progress and costs; (2) directly manufacture without paper drawings; (3) perform visualized stimulation and management; (4) quantify the construction progress by generating the update of evaluation and construction plans; (5) improve the quality of files and save the capital input on processes and management.

BIM based on digital graphic medium was applied in the construction phase of Balinghe Bridge [4-9].The authors and their teams applied the digital graphic medium based BIM technology in the installation of Balinghe Bridge. Skeleton grid modeling techniques are utilized; digital graphics are used as carrier of both geometric and physical attributes, and at the same time, motions and changes of graphical bodies are controlled by physical equations of the nature; graphical interaction based on physical equations can reflect the rules and results in reality, thus precise 3-D virtual reality models are established (see Fig.4); various proposals for installation of steel stiffening truss girder are simulated and optimized on the platform of virtual reality (see Fig.5).

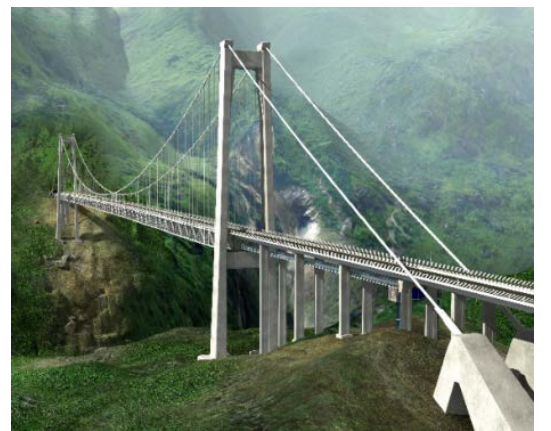

Fig.4 3D VR model of Balinghe Bridge

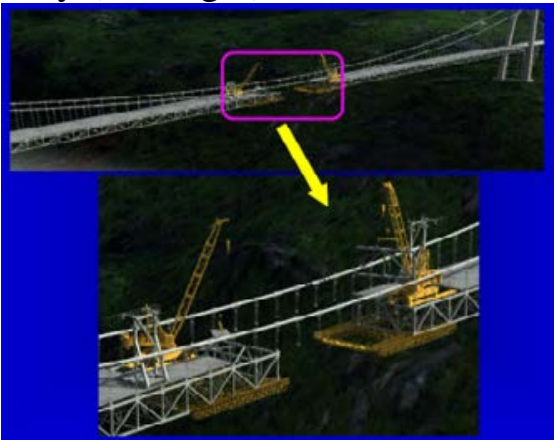

Fig.5 Simulation for installation of Balinghe Bridge

The entire installation of Balinghe Bridge was guided by the above described method. This has proposed a new design method, optimized and verified a new construction and installation method for building highways in western China, and provided strong technical support for a series of key techniques related to engineering design, construction and management. Significant economic benefits were brought about for improved management efficiency and reduced management costs.

\section{BIM Application on Parametric Family}

Based on digital graphic medium, parametric BIM family library was used to create a building project, and a 3-D BIM model was established by Revit (Fig.6-7).

Revit has functions of plan view and section view; the BIM model can be cut by the use of defined cut planes, thus the plan view of any cut plane can be obtained, marked with dimensions and exported out. See Fig.8.

Revit also has the function of HVAC modeling for the pipeline network and piping plans. Even a first-time user can use the intuitive layout and design tools to create 3-D models easily and effectively. By the use of built-in calculator, the dimensions of header pipe, branch pipes or even the entire system can be determined at one time. The model can be modified through movement or 
change of design elements on the screen in almost all views. Modification to any view will result in automatic changes to all model views and drawings, therefore, the software is able to provide accurate and consistent design results and files at all the time.

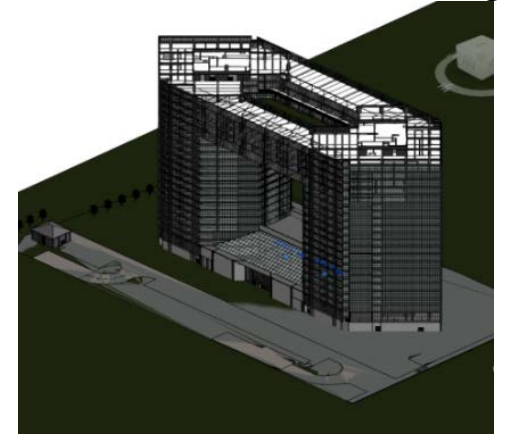

Fig.6 Revit building model

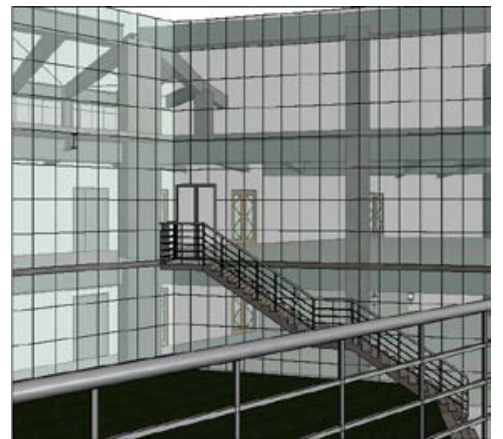

Fig.7 Revit curtain model

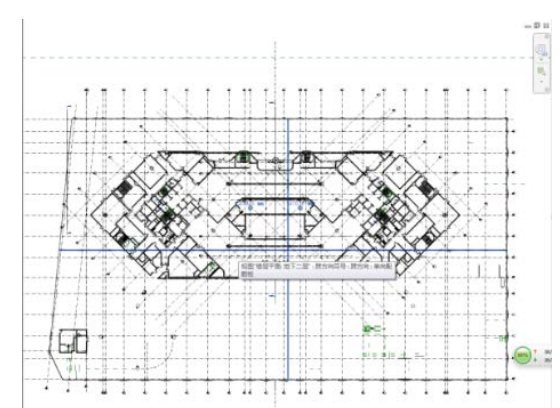

Fig.8 Revit building drawing

\section{BIM Application on ERP in Architecture}

BIM engineering basic data can be utilized for investment estimate, bill of quantities, bidding, contracting, determination of bidding subject, project budget, or construction cost control, material planning, project accounting and audit. Its multiple purpose features help achieve integrated management of material resources, financial resources and information resources (Fig.9).

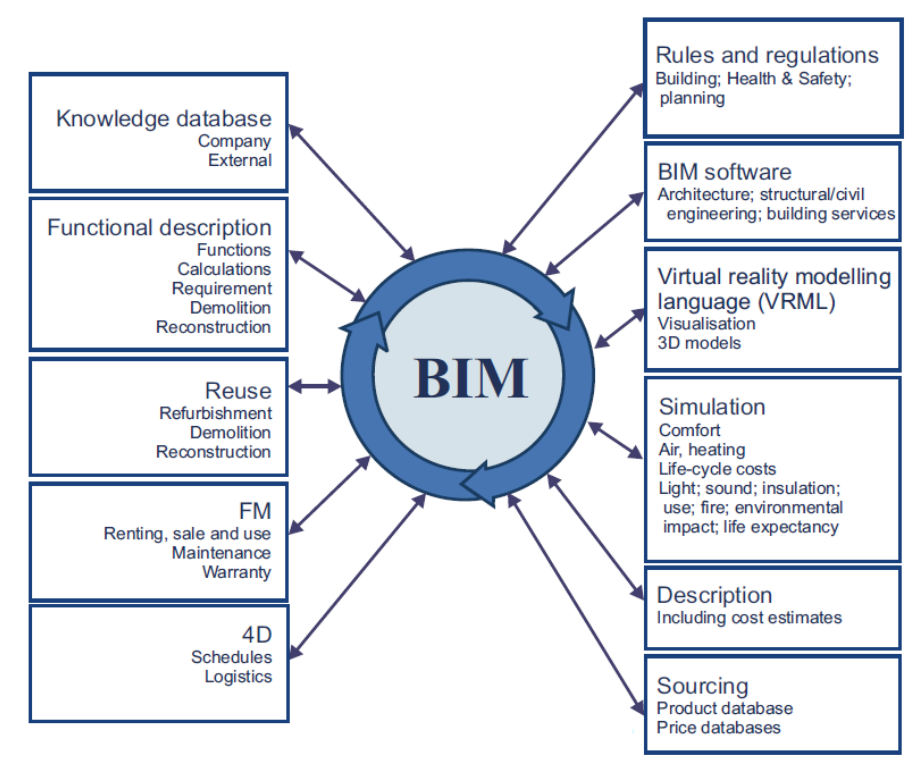

Fig.9 ERP element management from BIM[11]

\section{Conclusion}

The concept, construction method, description standard, basic theory and connotation of digital graphic medium are proposed in this paper, which forms corresponding projection and simulation patterns of the studied engineering object in the natural space and computer space, making the "graphics" become 5 dimensional information carrier with dynamic changing geometric and non-graphic attributes. The data of various specialties, periods and types are kept in step with digital graphics of the structure, providing an information sharing medium for different software and platforms; mechanical properties and movement pattern of the entire structure under different circumstances are following physical equations in the natural world, which are real-time simulated in virtual reality; remarkable results are achieved in engineering applications. Therefore, the developing and improving BIM technology will inevitably provide experience for other relevant fields of study. 


\section{Acknowledgement}

In this paper, the research was sponsored by Fund for Opening-up Cooperation Projects of Henan Province (142106000043), Fund for Key Science and Technology Breakthrough Projects of Henan Province (132102310037), Fund for Science and Technology Projects Jointly Taken by Chinese Academy of Sciences and Henan Province (112106000035), National Natural Science Foundation of China (No. 61471338), President Fund of UCAS, and Youth Innovation Promotion Association CAS (2015361).

\section{References}

[1] Wei Qun. Structural engineering visualization simulation methods and application [M]. Beijing: China Architecture \& Building Press, 2010.

[2] Wei Qun. Three-dimensional space structure graph slicing method based on the digital graphic medium: China, CN Patent 201310047748.8 [P]. 2012-02-28.

[3] Wei Qun. Three-dimensional space structure simulation based on the digital graphic medium: China, CN Patent 201210047628.8 [P]. 2012-02-28.

[4] Wei Qun, Jiang Hua, Peng Yundong and Peng Chengshan. Discussion about influence of joint rigidity of long span suspension bridge with stiffening steel truss girder on completed bridge working points [J]. Highway, 2009(03):29-34.

[5] Jiang Hua, Wei Qun, Peng Yundong. New construction techniques of large suspension bridge with steel truss girder of Baling River [J]. Journal of North China Institute of Water Conservancy and Hydroelectric Power, 2010(01):37-40.

[6] Wei Qun, Ji Guangkun, Yin Weibo. Secondary development of Inventor based on deep analysis method [J]. Journal of North China Institute of Water Conservancy and Hydroelectric Power, 2010(05):1-5.

[7] Yin Weibo, Wei Qun, Ji Guangkun. Implementation of automatic assembly technology based on BRep with Inventor [J]. Journal of North China Institute of Water Conservancy and Hydroelectric Power, 2010(06):23-26.

[8] Liu Shangwei, Yuan Dongmao, Tong Liang, Wei Qun. Three-dimensional modeling method of large steel bridge based on Inventor [J]. 2013(02):71-74.

[9] Wei Qun, Jiang Hua, Peng Yundong. Development and application of virtual reality visualization technology in bridge engineering [C]. 2009 Collection of Papers for National Construction Steel Structure Industry, 2009.

[10] Jiang Hua, Wei Qun, Peng Yundong. New construction techniques of large suspension bridge with steel truss girder of Baling River [J]. Journal of North China Institute of Water Conservancy and Hydroelectric Power, 2010, 31(1):37-40.

[11] Gupta A. Developing a BIM-based methodology to support renewable energy assessment of buildings [D]. Wales, UK: Cardiff School of Engineering, 2013. 